\title{
THE LATTICE OF COMPLETELY REGULAR SEMIGROUP VARIETIES
}

\author{
FRANCIS PASTIJN
}

(Received 12 December 1988)

Communicated by P. G. Trotter

\begin{abstract}
A completely regular semigroup is a semigroup which is a union of groups. The class CR of completely regular semigroups forms a variety. On the lattice $\mathcal{L}(\mathbf{C R})$ of completely regular semigroup varieties we define two closure operations which induce complete congruences. The consideration of a third complete congruence on $\mathcal{L}(\mathbf{C R})$ yields a subdirect decomposition of $\mathcal{L}(\mathbf{C R})$. Using these results we show that $\mathcal{L}(\mathbf{C R})$ is arguesian. This confirms the (tacit) conjecture that $\mathcal{L}(\mathbf{C R})$ is modular.
\end{abstract}

1980 Mathematics subject classification (Amer. Math. Soc.) (1985 Revision): primary 20 M 05;

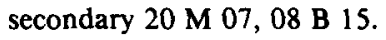

\section{Preliminaries}

Let $S$ be a semigroup equipped with a unary operation. Then $S$ is said to be a completely regular semigroup if $S$ satisfies, apart from the associative law, the identities

$$
x x^{-1} x=x, \quad x x^{-1}=x^{-1} x, \quad\left(x^{-1}\right)^{-1}=x .
$$

If this is the case, then $S$ is a union of groups: for $a \in S, a^{-1}$ is the inverse of $a$ within the unique maximal subgroup of $S$ to which $a$ belongs. Conversely, if $S$ is a semigroup which is a union of groups, then $S$ can be equipped with a unary operation ${ }^{-1}$ such that the resulting algebra of type $\langle 2,1\rangle$ satisfies the above identities. The class $\mathbf{C R}$ of completely reg-

(C) 1990 Australian Mathematical Society $0263-6115 / 90 \$ A 2.00+0.00$ 
ular semigroups thus forms a variety (i.e. an equational class). We shall investigate the lattice $\mathcal{L}(\mathbf{C R})$ of completely regular semigroup varieties.

The main sources of reference for this paper are [10], [13], [16]. We refer in particular to [13] and [16] for a more expanded bibliography concerning the study of completely regular semigroups from the point of view of universal algebra. We adopt the lattice theoretic terminology of [6]. As for a background on semigroup theory we refer to the standard texts [8], [14]. More details concerning the description of congruences on regular semigroups can be found in [10], [11] and [12]. We shall now supplement the existing notation with the following.

We list some subvarieties of $\mathbf{C R}$ which we shall need later. We first mention the notation for the variety under consideration, next the terminology for the completely regular semigroups which belong to the variety, and finally the identities which, in addition to the associative law and the identities for the variety of completely regular semigroups, determine the variety involved.

$\begin{array}{lll}\text { CS } & \text { completely simple semigroups } & x y(x y)^{-1} x=x, \\ \text { LG } & \text { left groups } & x y y^{-1}=x, \\ \text { RG } & \text { right groups } & x x^{-1} y=y, \\ \text { G } & \text { groups } & x x^{-1}=y y^{-1}, \\ \text { B } & \text { bands } & x^{2}=x, \\ \text { LRB } & \text { left regular bands } & x y x=x y, \\ \text { RRB } & \text { right regular bands } & x y x=y x, \\ \text { LNB } & \text { left normal bands } & x^{2}=x, x y z=x z y, \\ \text { RNB } & \text { right normal bands } & x^{2}=x, x y z=y x z, \\ \text { SL } & \text { semilattices } & x^{2}=x, x y=y x, \\ \text { RB } & \text { rectangular bands } & x y x=x, \\ \text { LZ } & \text { left zero semigroups } & x y=x, \\ \text { RZ } & \text { right zero semigroups } & x y=y, \\ \text { T } & \text { trivial semigroups } & x=y .\end{array}$

From the description of the lattice of band varieties in [1], [3] or [4] and from [17] it follows that the above-mentioned completely regular semigroup varieties generate the finite lattice of Figure 1. Further details concerning the structure of the completely regular semigroups which belong to either of the mentioned varieties can be found in [14] or [15].

If $\mathbf{V}$ and $\mathbf{W}$ are subvarieties of $\mathbf{C R}$, then $\mathbf{V} \circ \mathbf{W}$ will denote the class of all completely regular semigroups $S$ on which one can define a congruence $\rho$ such that $S / \rho \in \mathrm{W}$ and such that every $\rho$-class which forms a completely regular semigroup belongs to $\mathbf{V}$. The class $\mathbf{V} \circ \mathbf{W}$ is said to be the $\mathrm{Mal}^{\prime}$ cev product of $\mathbf{V}$ and $\mathbf{W}$ (in $\mathbf{C R}$ ). We refer to [9] for more details concerning the $\mathrm{Mal}^{\prime}$ cev product of completely regular semigroup varieties. 


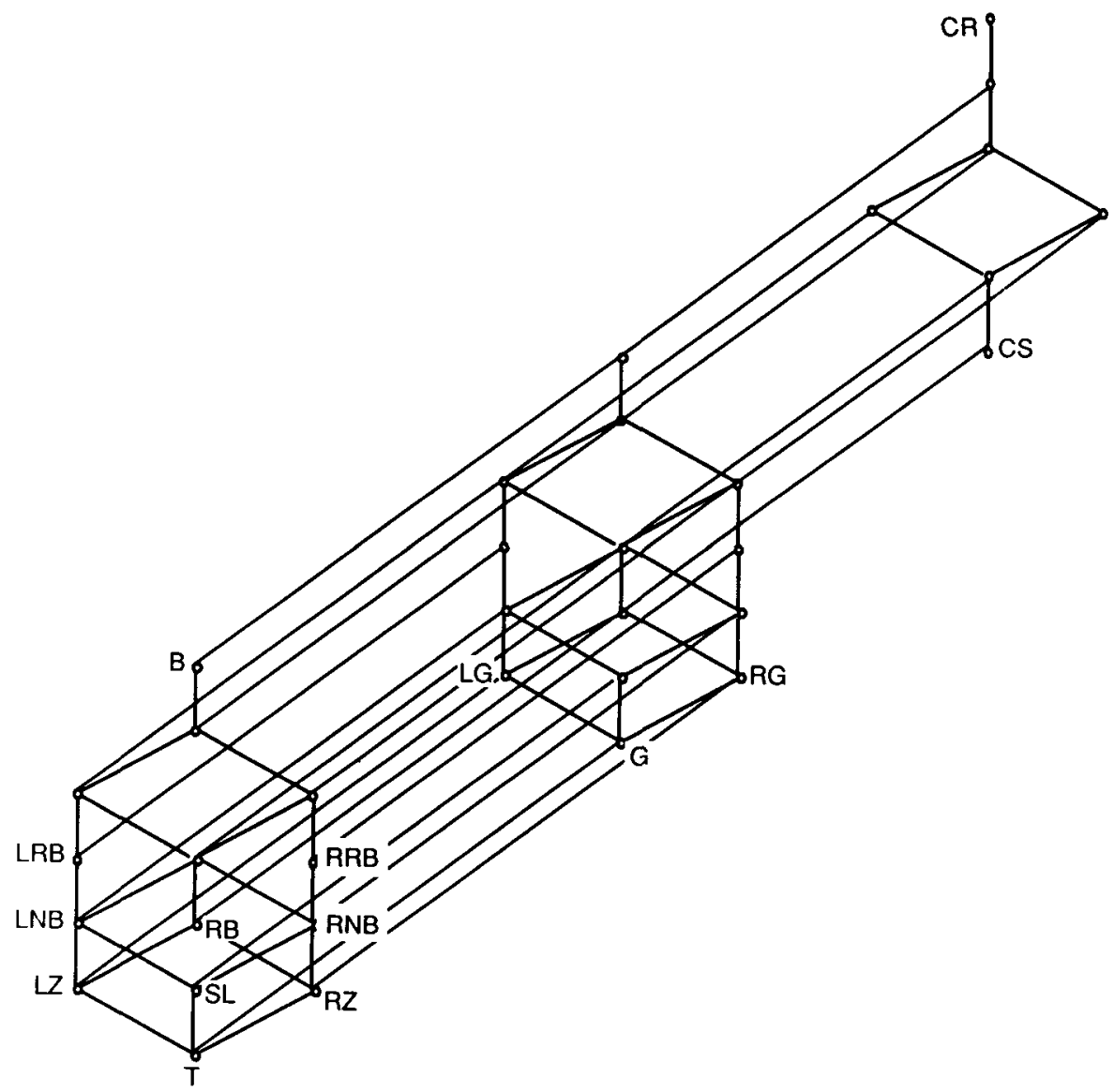

Figure 1

In the following $X$ will be a countably infinite set and $U(X)$ the free unary semigroup on the set $X$. If $\mathbf{V}$ is any variety of unary semigroups, then $\rho_{\mathbf{V}}$ will denote the least congruence on $U(X)$ such that $U(X) / \rho_{\mathbf{y}} \in \mathbf{V}$. We let $\mathcal{L}$ be the lattice of fully invariant congruences on $U(X)$ which contain $\rho_{\mathrm{CR}}$. For any $\rho \in \mathcal{L}, U(X) / \rho$ generates a variety of completely regular semigroups, which we shall denote by $V_{\rho}$. It is well-known that

$$
\mathcal{L} \rightarrow \mathcal{L}(\mathbf{C R}), \quad \rho \rightarrow \mathbf{V}_{\rho}
$$

and

$$
\mathcal{L}(\mathbf{C R}) \rightarrow \mathcal{L}, \quad \mathbf{V} \rightarrow \rho_{\mathbf{v}}
$$

are mutually inverse antiisomorphisms.

One often considers $U(X)$ to be a subset of the free monoid $F$ on the set $X \cup\left\{(,)^{-1}\right\}$. For any $a \in U(X)$, with $a=b c$ in $F$, one says that $b[c]$ 
is an initial [final] segment of $a$. We then denote by $\hat{b}$ and $\hat{c}$ the elements of $U(X)$ obtained from $b$ and $c$ by deleting unmatched parentheses.

If $a \in F$, then the content of $a$, in notation $c(a)$, is the set of the variables (i.e. elements of $X$ ) which occur in $a$. If $b$ is the largest initial segment of $a \in U(X)$ in $|\mathbf{c}(a)|-1$ variables, then we put $\mathbf{s}(a)=\hat{b}$; dually, if $c$ is the largest terminal segment of $a$ in $|c(a)|-1$ variables, then we put $\mathbf{d}(a)=\hat{c}$.

\section{Two complete endomorphisms of $\mathcal{L}(\mathbf{C R})$}

For any regular semigroup $S$, we denote by $E(S)$ the set of idempotents of $S$. For $e, f \in E(S)$ we put $e \leq_{l} f$ if and only if $e f=e$. The relation $\leq_{r}$ on $E(S)$ is defined in a dual way. We recall the following result.

Result 1 [10, Theorem 15]. Let $S$ be a regular semigroup. The following are equivalent for congruences $\rho$ and $\theta$ on $S$ :

(i) in the lattice of equivalence relations on $S$ we have

$$
\rho \vee R=\theta \vee R,
$$

$$
\rho \cap \leq_{l}=\theta \cap \leq_{l} .
$$

For any $\rho, \theta \in \mathcal{L}$ we put $\rho T_{r} \theta$ if and only if

$$
\rho / \rho_{\mathbf{C R}} \vee R=\theta / \rho_{\mathbf{C R}} \vee R
$$

where $R$ stands for Green's $R$-relation on the free completely regular semigroup $U(X) / \rho_{\mathbf{C R}}$. By the above Result $1,(1)$ is equivalent with

$$
\rho / \rho_{\mathrm{CR}} \cap \leq_{l}=\theta / \rho_{\mathrm{CR}} \cap \leq_{l},
$$

where $\leq_{l}$ is the appropriate relation on the set of idempotents of $U(X) / \rho_{\mathbf{C R}}$. It readily follows that $T_{r}$ is a complete congruence on $\mathcal{L}$.

If $\alpha$ is any binary relation on the free completely regular semigroup $U(X) / \rho_{\mathbf{C R}}$, then $\alpha^{*}$ denotes the least congruence containing $\alpha$. If $\alpha$ is an equivalence relation, then $\alpha^{\circ}$ denotes the greatest congruence contained in $\alpha$.

In the following theorem we use $R$ and $\leq_{l}$ as in (1) and (2).

THEOREM 2. For any $\rho \in \mathcal{L}$, the $T_{r}$-class $\rho T_{r}$ of $\rho$ is an interval $\left[\rho_{T_{r}}, \rho^{T_{r}}\right]$, where $\rho_{T}$, and $\rho^{T_{r}}$ are given by

$$
\rho_{T_{r}} / \rho_{\mathrm{CR}}=\left(\rho / \rho_{\mathrm{CR}} \cap \leq_{l}\right)^{*} \text { and } \rho^{T_{r}} / \rho_{\mathrm{CR}}=\left(\rho / \rho_{\mathrm{CR}} \vee R\right)^{\circ} \text {. }
$$


Proof. Following [10, Corollary 6], $\left(\rho / \rho_{\mathrm{CR}} \cap \leq_{l}\right)^{*}$ is the least among the congruences $\alpha$ on $U(X) / \rho_{\mathrm{CR}}$ which satisfy

$$
\rho / \rho_{\mathrm{CR}} \cap \leq_{l}=\alpha \cap \leq_{l} .
$$

Since $\rho / \rho_{\mathrm{CR}} \cap \leq_{l}$ is invariant under every endomorphism of $U(X) / \rho_{\mathrm{CR}}$, then so is $\left(\rho / \rho_{\mathbf{C R}} \cap \leq_{l}\right)^{*}$. Hence there exists a (unique) $\rho_{T_{r}} \in \mathcal{L}$ such that

$$
\left(\rho / \rho_{\mathbf{C R}} \cap \leq_{l}\right)^{*}=\rho_{T_{r}} / \rho_{\mathbf{C R}},
$$

and $\rho_{T_{r}}$ is the smallest element in $\rho T_{r}$.

Following [10, Corollary 16], $\left(\rho / \rho_{\mathrm{CR}} \vee R\right)^{\circ}$ is the greatest among the congruences $\alpha$ on $U(X) / \rho_{\mathbf{C R}}$ which satisfy

$$
\rho / \rho_{\mathbf{C R}} \vee R=\alpha \vee R \text {. }
$$

Since the equivalence relation $\rho / \rho_{\mathbf{C R}} \vee R$ is invariant under every endomorphism, and since $X$ is countably infinite, it follows that $\left(\rho / \rho_{\mathbf{C R}} \vee R\right)^{\circ}$ is a fully invariant congruence on $U(X) / \rho_{\mathbf{C R}}[13$, Lemma 2.3]. Hence there

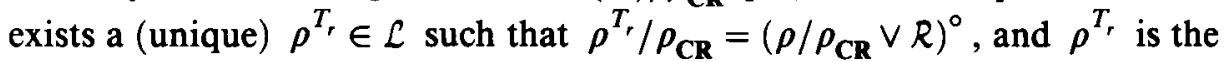
greatest element in $\rho T_{r}$.

In the following we characterize the $\rho_{T_{r}}$ in another way.

LEMMA 3. For any $\mathbf{V} \in \mathcal{L}(\mathbf{C R}),\left(\rho_{\mathbf{v}}\right)_{T_{r}}=\rho_{\mathbf{R G o V}}$.

Proof. Immediate from [10, Theorem 15] and the results of $[9, \S 3]$.

Before we give another characterization of the $\rho^{T_{r}}$, we have the following.

Corollary 4. For $\mathbf{V}, \mathbf{W} \in \mathcal{L}(\mathbf{C R})$,

$$
\rho_{\mathbf{V}} \quad T_{r} \rho_{\mathbf{W}} \Leftrightarrow \mathbf{R G} \circ \mathbf{V}=\mathbf{R G} \circ \mathbf{W} .
$$

LEMMA 5. (i) In $\mathcal{L} / T_{r}$ we have that $\rho_{\mathrm{T}} T_{r}, \rho_{\mathrm{LZ}} T_{r}, \rho_{\mathrm{SL}} T_{r}, \rho_{\mathrm{LNB}} T_{r}$ and $\rho_{\mathrm{LRB}} T_{r}$ form the principal filter of Figure 2.

(ii) The $T_{r}$-classes considered in (i) are the intervals

$$
\begin{gathered}
\rho_{\mathrm{T}} T_{r}=\left[\rho_{\mathrm{RG}}, \rho_{\mathrm{T}}\right], \quad \rho_{\mathbf{L Z}} T_{r}=\left[\rho_{\mathrm{CS}}, \rho_{\mathrm{LZ}}\right], \\
\rho_{\mathrm{SL}} T_{r}=\left[\rho_{\mathrm{RG} \circ \mathrm{SL}}, \rho_{\mathrm{SL}}\right], \quad \rho_{\mathbf{L N B}} T_{r}=\left[\rho_{\mathrm{RGoLNB}}, \rho_{\mathrm{LNB}}\right], \\
\rho_{\mathrm{LRB}} T_{r}=\left[\rho_{\mathrm{RGoLRB}}, \rho_{\mathbf{L R B}}\right] .
\end{gathered}
$$

(iii) If $\rho \in \mathcal{L}$, then

$$
\rho_{\text {RGoLNB }} \nsubseteq \rho \Leftrightarrow \rho \subseteq \rho_{\text {LRB }}
$$

Proof. (i) Let $\rho_{\mathrm{LRB}} T_{r} \leq \theta T_{r}$ for some $\theta \in \mathcal{L}$. Let us put $\rho=\rho_{\mathrm{LRB}} \vee \theta$. 


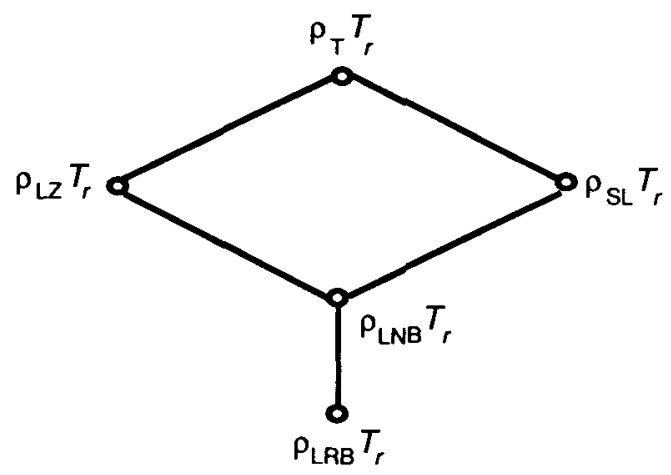

Figure 2

Since $T_{r}$ is a congruence we have that $\rho T_{r}=\theta T_{r}$, where $\mathbf{V}_{\rho} \subseteq \mathbf{L R B}$. From [1], [3], [4] or [14] we have that $V_{\rho}$ is either one of the varieties LRB , LNB, SL, LZ or T. Since these varieties form a 5-element lattice which is dually isomorphic to the lattice of Figure 2, it now suffices to show that the fully invariant congruences associated with these five varieties are in different $T_{r}$-classes. This is obviously true by Corollary 4 and the fact that RG $\circ \mathbf{L R B}, \mathbf{R G} \circ \mathbf{L N B}, \mathbf{R G} \circ \mathbf{S L}, \mathbf{C S}=\mathbf{R G} \circ \mathbf{L Z}$ and RG are different varieties.

(ii) If $\rho \in \mathcal{L}$ and $\rho_{\mathrm{LRB}} \subset \rho$, then we have, as in (i) that $\rho=\rho_{\mathrm{T}}, \rho=$ $\rho_{\mathrm{LZ}}, \rho=\rho_{\mathrm{SL}}$ or $\rho=\rho_{\mathrm{LNB}}$. Since by (i) none of these congruences belong to $\rho_{\mathrm{LRB}} T_{r}$, we conclude that $\rho_{\mathrm{LRB}}$ is the greatest element in its $T_{r}$-class. In a similar way one shows that $\rho_{\mathrm{T}}, \rho_{\mathrm{LZ}}, \rho_{\mathrm{SL}}$ and $\rho_{\mathrm{LNB}}$ are the greatest in their respective $T_{r}$-classes. The remaining statements in (ii) follow from Lemma 3.

(iii) If $\rho \in \mathcal{L}$ and $\rho \subseteq \rho_{\mathrm{LRB}}$, then $\rho T_{r} \leq \rho_{\mathrm{LRB}} T_{r}<\rho_{\mathrm{LNB}} T_{r}$. Since $\rho_{\mathrm{RGoLNB}}$ is the smallest element of $\rho_{\mathrm{LNB}}$, this entails that $\rho_{\mathrm{RGoLNB}} \nsubseteq \rho$.

Let $\rho \in \mathcal{L}$ and $\rho_{\text {RGoLNB }} \notin \rho$; that is, $\mathbf{V}_{\rho} \nsubseteq \mathbf{R G} \circ \mathbf{L N B}$. By [15, Theorem 8] the variety $\mathbf{V}_{\rho}$ contains a 2-element left zero semigroup with an identity adjoined. Since this 3-element semigroup generates LRB, we have that $\mathbf{L R B} \subseteq \mathrm{V}_{\rho}$, whence $\rho \subseteq \rho_{\mathrm{LRB}}$.

From Lemma 5 it follows that in $\mathcal{L} / T_{r}$ every $\rho T_{r}$ is comparable with $\rho_{\mathrm{LRB}} T_{r}$.

For $\rho \in \mathcal{L}$ we define the relation $\rho_{0}$ on the free unary semigroup $U(X)$ by putting $a \rho_{0} b$ if and only if for some $c, d \in U(X)$ we have $c \rho d, a=\mathbf{s}(c)$ and $b=\mathbf{s}(d)$. This relation $\rho_{0}$ was introduced by Polák in $[16, \S 1]$.

LEMMA 6. For $\rho \in \mathcal{L}$, with $\rho \subseteq \rho_{\mathrm{SL}}$ we have

$$
\rho_{0} \notin \rho_{\mathbf{S L}}, \quad \mathbf{L Z} \subseteq \mathbf{V}_{\rho} \Leftrightarrow \rho T_{r} \rho_{\mathbf{L N B}},
$$


and

$$
\rho_{0}=U(X) \times U(X) \Leftrightarrow \mathbf{L Z} \nsubseteq \mathbf{V}_{\rho} \Leftrightarrow \rho T_{r} \rho_{\mathrm{SL}}
$$

Proof. If $\rho_{0} \nsubseteq \rho_{\mathrm{SL}}$ and $\mathbf{L Z} \subseteq \mathbf{V}_{\rho}$, then by [16, Lemma 6] $\mathbf{V}_{\rho}$ is contained in the variety of $\mathcal{L}(\mathbf{C R})$ which is determined by the identity

$$
x y z=(x z y)(x z y)^{-1} x y z,
$$

and by [15, Theorem 8] this variety is exactly $\mathbf{R G} \circ \mathbf{L N B}$. Since $\mathbf{L Z} \subseteq \mathbf{v}_{\rho}$ we know that $\mathbf{V}_{\rho} \notin[\mathbf{S L}, \mathbf{R G} \circ \mathbf{S L}]$. Hence

$$
\rho_{\mathrm{RGoLNB}} \subseteq \rho \subseteq \rho_{\mathrm{SL}}, \quad \rho \notin\left[\rho_{\mathrm{RGoSL}}, \rho_{\mathrm{SL}}\right],
$$

and by Lemma 5 we can conclude that $\rho T_{r} \rho_{\mathrm{LNB}}$.

If $\rho T_{r} \rho_{\mathbf{L N B}}$, then $\mathbf{L N B} \subseteq \mathbf{V}_{\rho} \subseteq \mathbf{R G} \circ \mathbf{L N B}$ by Lemma 5 , and from this we have already that $\mathbf{L Z} \subseteq \mathbf{V}_{\rho}$. Further, by [15, Theorem 8] $\mathbf{V}_{\rho}$ satisfies the identity (3). Hence by [16, Lemma 6] we also have $\rho_{0} \notin \rho_{\mathrm{SL}}$.

If $\mathbf{L Z} \nsubseteq \mathbf{V}_{\rho}$, then by [15, Theorem 9] $\mathbf{V}_{\rho}$ consists of semilattices of right groups. Thus $\rho_{\text {RGoSL }} \subseteq \rho \subseteq \rho_{\text {SL }}$, which by Lemma 5 implies that $\rho T_{r} \rho_{\mathrm{SL}}$. Conversely, if $\rho T_{r} \rho_{\mathrm{SL}}$, then $\rho_{\mathrm{RGoSL}} \subseteq \rho$. By [15, Theorem 9] the completely regular semigroups which belong to $\mathbf{R G} \circ \mathbf{S L}$ are determined by the fact that their idempotents form a right regular band. Hence LZ $\mathbb{Z}$ $\mathbf{R G} \circ \mathbf{S L}$ and a fortiori $\mathbf{L Z} \nsubseteq \mathbf{V}_{\rho}$. The remaining part of the statement is the content of [16, Lemma 5].

LEMMA 7. If $\rho \in \mathcal{L}$ with $\rho \subseteq \rho_{\mathrm{LRB}}$, then $\rho_{0}=\rho^{T}$.

Proof. Let $a \rho^{T_{r}} b$ for some $a, b \in U(X)$. Since $\rho \subseteq \rho_{\text {LRB }}$ we have that $\rho T_{r} \leq \rho_{\mathrm{LRB}} T_{r}<\rho_{\mathrm{SL}} T_{r}$, whence $\rho^{T_{r}} \subset \rho_{\mathrm{SL}}$. Therefore $\mathrm{c}(a)=\mathfrak{c}(b)$ [2]. We choose $x \notin \mathbf{c}(a)=\mathbf{c}(b)$. Since $a x \rho^{T_{r}} b x$, we have by [10, Theorem 15] that $(a x) \rho R(b x) \rho$ in $U(X) / \rho$, and so $a x(a x)^{-1} b x \rho b x$. Further, $a=\mathbf{s}\left(a x(a x)^{-1} b x\right)$ and $b=\mathbf{s}(b x)$. Thus $a \rho_{0} b$ and we conclude that $\rho^{T_{r}} \subseteq \rho_{0}$.

Let $a \rho_{0} b$. By Lemmas 5 and 6 we have that $\rho_{0} \subseteq \rho_{\mathrm{SL}}$, and thus $\mathbf{c}(a)=\mathbf{c}(b)$ [2]. By [16, Theorem 3(2)] there exist $c, d \in U(X)$ such that $c \rho d$ and $\mathbf{s}(a)=\mathbf{s}(c), \mathbf{s}(b)=\mathbf{s}(d)$. We define $x, y, x^{\prime}, y^{\prime} \in X$ by

$$
\begin{array}{ll}
\{x\}=\mathbf{c}(a)-\mathbf{c}(\mathbf{s}(a)), & \{y\}=\mathbf{c}(b)-\mathbf{c}(\mathbf{s}(b)), \\
\left\{x^{\prime}\right\}=\mathbf{c}(c)-\mathbf{c}(\mathbf{s}(c)), & \left\{y^{\prime}\right\}=\mathbf{c}(d)-\mathbf{c}(\mathbf{s}(d)) .
\end{array}
$$

Since $c \rho d$ and $\rho \subseteq \rho_{\mathrm{SL}}$ we have that $\mathbf{c}(c)=\mathbf{c}(d)$ [2]. Thus if $\mathbf{c}(\mathbf{s}(a)) \neq$ $\mathbf{c}(\mathbf{s}(b))$, then $x=x^{\prime} \in \mathbf{s}(b)$ and $y=y^{\prime} \in \mathbf{s}(a)$. If $\mathbf{c}(\mathbf{s}(a))=\mathbf{c}(\mathbf{s}(b))$, then we 
can assume that $x=x^{\prime}=y=y^{\prime}$. In either of the two cases we have from $[2, \S 5]$ that

$$
\begin{gathered}
a \rho_{\mathbf{C R}} R(\mathbf{s}(a) x) \rho_{\mathbf{C R}}=(\mathbf{s}(c) x) \rho_{\mathbf{C R}} R\left(c \rho_{\mathbf{C R}}\right) \rho / \rho_{\mathbf{C R}}\left(d \rho_{\mathbf{C R}}\right), \\
R(\mathbf{s}(d) y) \rho_{\mathbf{C R}}=(\mathbf{s}(b) y) \rho_{\mathbf{C R}} R b \rho_{\mathbf{C R}}
\end{gathered}
$$

where $R$ stands for Green's $R$-relation on the free completely regular semigroup $U(X) / \rho_{\mathrm{CR}}$. Hence $\rho_{0} / \rho_{\mathrm{CR}} \subseteq \rho / \rho_{\mathrm{CR}} \vee R$, and, since $\rho_{0} / \rho_{\mathrm{CR}}$ is a congruence on $U(X) / \rho_{\mathrm{CR}}$ by [16, Theorem $\left.3(4)\right]$, we have from Theorem 2 that

$$
\rho_{0} / \rho_{\mathrm{CR}} \subseteq\left(\rho / \rho_{\mathrm{CR}} \vee R\right)^{\circ}=\rho^{T_{r}} / \rho_{\mathrm{CR}},
$$

and thus $\rho_{0} \subseteq \rho^{T_{r}}$. We conclude that $\rho_{0}=\rho^{T_{r}}$.

We are now ready for one of the main results of this section.

THEOREM 8. The closure operation $\rho \rightarrow \rho^{T_{r}}$ is a complete idempotent endomorphism of $\mathcal{L}$ which induces the complete congruence $T_{r}$.

Proof. Let $\left(\rho_{i}, i \in I\right)$ be a family of elements of $\mathcal{L}$. Then

$$
\bigcap_{i \in I} \rho_{i}^{T_{r}}=\left(\bigcap_{i \in I} \rho_{i}\right)^{T_{r}}
$$

since $T_{r}$ is a complete congruence on $\mathcal{L}$. If for some $i \in I, \rho_{i} \nsubseteq$ $\rho_{\text {LRB }}$, then by Lemma $5, \rho_{\text {RGoLNB }} \subseteq \rho_{i}$ so that $\rho_{i}^{T_{r}}$ is one of the relations $\rho_{\mathrm{LNB}}, \rho_{\mathrm{SL}}, \rho_{\mathrm{LZ}}$ or $\rho_{\mathrm{T}}$. Using Lemma 5 we can now easily conclude that

$$
\bigvee_{i \in I} \rho_{i}^{T_{r}}=\left(\bigvee_{i \in I} \rho_{i}\right)^{T_{r}} .
$$

We now set out to prove (5) in case $\rho_{i} \subseteq \rho_{\mathbf{L R B}}$ for all $i \in I$. Then $\bigvee_{i \in I} \rho_{i} \subseteq \rho_{\mathbf{L R B}}$, and so by Lemma 7 ,

$$
\left(\bigvee_{i \in I} \rho_{i}\right)^{T_{r}}=\left(\bigvee_{i \in I} \rho_{i}\right)_{0}
$$

Let $a\left(\bigvee_{i \in I} \rho_{i}\right)^{T_{r}} b$ for some $a, b \in U(X)$. By the above there exist $c, d \in$ $U(X)$ such that $a=\mathbf{s}(c), b=\mathbf{s}(d)$ and $c\left(V_{i \in I} \rho_{i}\right) d$. There exist $n \in \mathbf{N}$, $q_{0}, \ldots, q_{n} \in U(X)$ and $\rho_{i_{1}}, \ldots, \rho_{i_{n}}$ with $i_{1}, \ldots, i_{n} \in I$ such that

$$
c=q_{0} \rho_{i_{1}} q_{1} \ldots q_{n-1} \rho_{i_{n}} q_{n}=d
$$

Thus,

$$
a=\mathbf{s}(c)=\mathbf{s}\left(q_{0}\right)\left(\rho_{i_{1}}\right)_{0} \mathbf{s}\left(q_{1}\right) \ldots \mathbf{s}\left(q_{n-1}\right)\left(\rho_{i_{n}}\right)_{0} \mathbf{s}\left(q_{n}\right)=\mathbf{s}(d)=b .
$$


Again by Lemma 7, $\left(\rho_{i_{j}}\right)_{0}=\left(\rho_{i_{j}}\right)^{T_{r}}$ for $j=1, \ldots, n$. Hence $a\left(\bigvee_{i \in I} \rho_{i}^{T_{r}}\right) b$. We conclude that

$$
\left(\bigvee_{i \in I} \rho_{i}\right)^{T_{r}} \subseteq \bigvee_{i \in I} \rho_{i}^{T_{r}}
$$

The reverse inclusion holds since $T_{r}$ is a complete congruence. Therefore (5) is satisfied.

From (4) and (5) we see that the elements $\rho^{T_{r}}$ of $\mathcal{L}$ form a complete sublattice. From this the remaining part of the statement of the theorem can be proved easily.

Because of the anti-isomorphism $\rho \rightarrow \mathbf{v}_{\rho}$ of $\mathcal{L}$ onto $\mathcal{L}(\mathbf{C R})$, the situation described in Theorem 8 carries over to $\mathcal{L}(\mathbf{C R})$. We thus obtain a complete congruence on $\mathcal{L}(\mathbf{C R})$ which we shall again denote by $T_{r}$.

THEOREM 9. The relation $T_{r}$ which is given by

$$
\mathbf{V} T_{r} \mathbf{W} \Leftrightarrow \mathbf{R G} \circ \mathbf{V}=\mathbf{R G} \circ \mathbf{W} \quad(\mathbf{V}, \mathbf{W} \in \mathcal{L}(\mathbf{C R}))
$$

is a complete congruence on $\mathcal{L}(\mathbf{C R})$. For $\mathbf{V} \in \mathcal{L}(\mathbf{C R})$ the $T_{r}$-class $\mathbf{V} T_{r}$ is an interval $\left[\mathbf{V}_{T_{r}}, \mathbf{V}^{T_{r}}\right]$, where $\mathbf{V}^{T_{r}}=\mathbf{R G} \circ \mathbf{V}$. The $\mathbf{V}_{T_{r}}(\mathbf{V} \in \mathcal{L}(\mathbf{C R}))$ form a complete sublattice of $\mathcal{L}(\mathbf{C R})$. The dual closure operation $\mathbf{V} \rightarrow \mathbf{V}_{T_{r}}$ is a complete idempotent endomorphism of $\mathcal{L}(\mathbf{C R})$ which induces $T_{r}$.

Proof. Immediate from Lemma 3, Corollary 4 and Theorem 8.

If $\mathbf{V} \in \mathcal{L}(\mathbf{C R})$, then $\mathbf{R G} \circ \mathbf{V}$ is the greatest element $\mathbf{V}^{T_{r}}$ in the $T_{r}$-class of V. As in [9, Proposition 6.4] one can derive a set of defining identities for $\mathbf{V}^{T_{r}}$ from a set of defining identities for $\mathbf{V}$. The fully invariant congruence associated with $\mathbf{V}_{T_{r}}$ is $\left(\rho_{\mathrm{V}}\right)^{T_{r}}$ and is given by either Lemma 5 or Lemma 7 .

Obviously the notions and results obtained so far in this section have their left-right duals. The congruence on $\mathcal{L}$ or $\mathcal{L}(\mathbf{C R})$ which is the dual of $T_{r}$ will be denoted by $T_{l}$. The dual closure operation $\mathbf{V} \rightarrow \mathbf{V}_{T_{l}}$ is the other complete endomorphism of $\mathcal{L}(\mathbf{C R})$ referred to in the title of this section. The relation $T=T_{l} \cap T_{r}$ is again a complete congruence and the $T$-classes are intervals

$$
\rho T=\left[\rho_{T}, \rho^{T}\right], \quad \mathbf{V} T=\left[\mathbf{V}_{T}, \mathbf{V}^{T}\right] \quad(\rho \in \mathcal{L}, \mathbf{V} \in \mathcal{L}(\mathbf{C R})),
$$

where

$$
\begin{gathered}
\rho_{T}=\rho_{T_{l}} \vee \rho_{T_{r}}, \quad \rho^{T}=\rho^{T_{l}} \cap \rho^{T_{r}}, \quad \mathbf{V}_{T}=\mathbf{V}_{T_{l}} \vee \mathbf{V}_{T_{r}}, \\
\mathbf{V}^{T}=\mathbf{V}^{T_{l}} \cap \mathbf{V}^{T_{r}}=\mathbf{L G} \circ \mathbf{V} \cap \mathbf{R G} \circ \mathbf{V}=\mathbf{G} \circ \mathbf{V},
\end{gathered}
$$




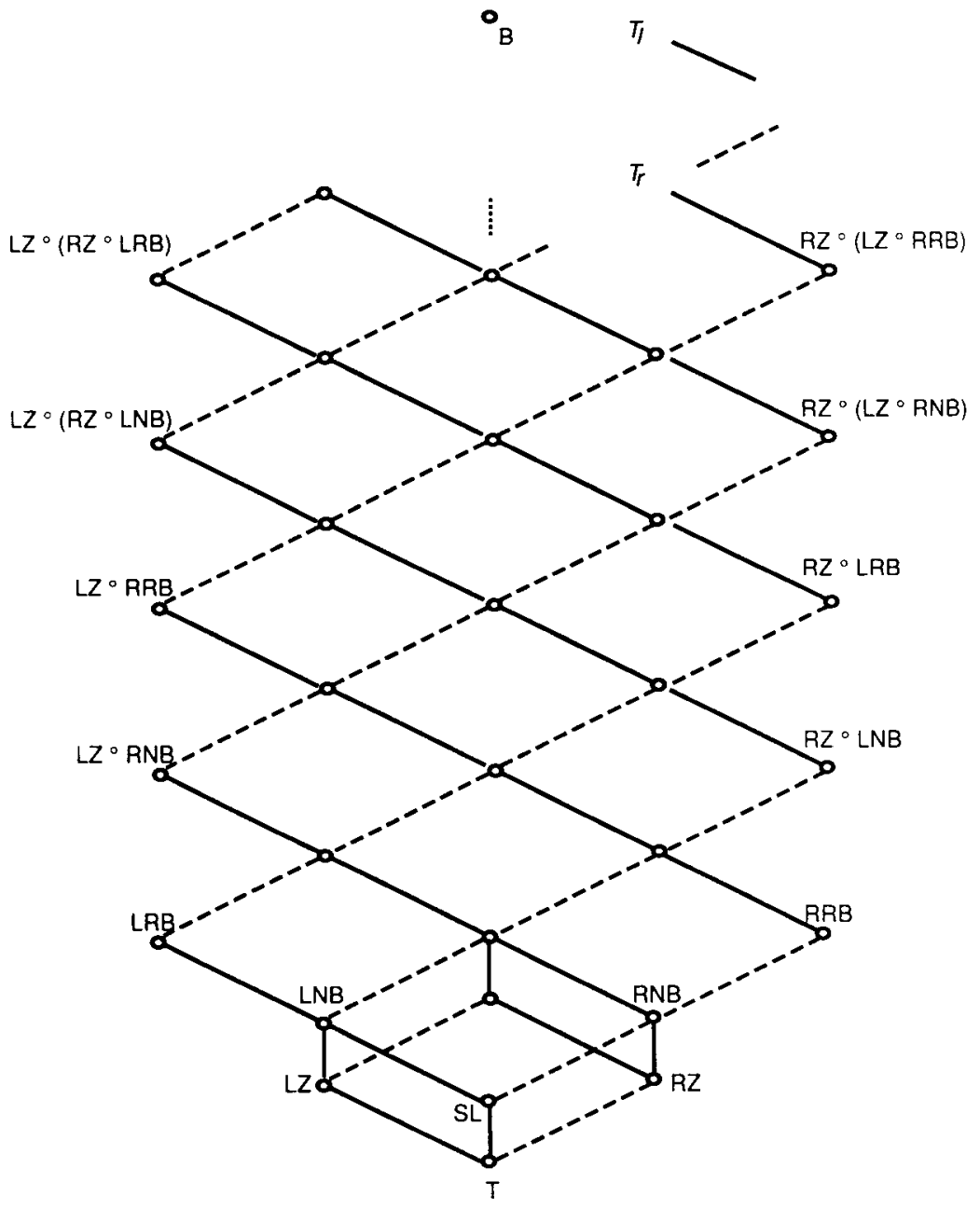

Figure 3

where the last equality stems from [9]. From this it also follows that the relation $T$ on $\mathcal{L}(\mathbf{C R})$ can be defined by

$$
\mathbf{V} T \mathbf{W} \Leftrightarrow \mathbf{G} \circ \mathbf{V}=\mathbf{G} \circ \mathbf{W} \text {. }
$$

The variety $\mathbf{V}^{T}$ was denoted by $\mathbf{V}_{\left(\rho_{\mathbf{v}}\right)_{\min }}$ in [13] and by $\mathbf{V}^{+}$in [19]. Using [9, Proposition 6.4] or [19, Theorem 3.9] we can derive a set of defining identities for $\mathbf{V}^{T}$ from a set of defining identities for $\mathbf{V}$.

EXAMPLE 10. If $\mathbf{V}$ and $\mathbf{W}$ are band varieties then from Theorem 9 and its dual we have that

$$
\mathbf{V} T_{r} \mathbf{W} \Leftrightarrow \mathbf{R Z} \circ \mathbf{V}=\mathbf{R Z} \circ \mathbf{W},
$$




$$
\begin{gathered}
\mathbf{V} T_{l} \mathbf{W} \Leftrightarrow \quad \mathbf{L Z} \circ \mathbf{V}=\mathbf{L Z} \circ \mathbf{W}, \\
\mathbf{V} T \mathbf{W} \Leftrightarrow \quad \mathrm{V}=\mathbf{W} .
\end{gathered}
$$

Therefore $T=T_{l} \cap T_{r}$ induces the equality on the lattice $\mathcal{L}(\mathbf{B})$ of band varieties. This yields a subdirect decomposition of $\mathcal{L}(\mathbf{B})$. According to Theorem 9, the $\mathbf{V}_{T_{r}}(\mathbf{V} \in \mathcal{L}(\mathbf{B}))$ form a complete sublattice of $\mathcal{L}(\mathbf{B})$ which is isomorphic to $\mathcal{L}(\mathbf{B}) /\left(\left.T_{r}\right|_{\mathcal{L}(\mathbf{B})}\right)$. Using the above and the results of [1], [3] or [4] we find that this lattice consists of the elements T, LZ, SL, B and the elements of the chain $C_{r}$ which can be defined recursively as follows (see Figure 3):

(i) LNB, LRB, LZ $\circ \mathbf{R N B}, \mathbf{L Z} \circ \mathbf{R R B}$ are in $C_{r}$,

(ii) if $\mathbf{V} \in \mathcal{C}_{r}$, then $\mathbf{L Z} \circ(\mathbf{R Z} \circ \mathbf{V}) \in \mathcal{C}_{r}$,

(iii) $C_{r}$ is the smallest sublattice of $\mathcal{L}(\mathbf{B})$ satisfying (i) and (ii).

Therefore $\mathcal{L}(\mathbf{B}) /\left(\left.T_{r}\right|_{\mathcal{L}(\mathbf{B})}\right)$ is isomorphic to the lattice of Figure 4 . By leftright duality, also $\mathcal{L}(\mathbf{B}) /\left(\left.T_{l}\right|_{\mathcal{L}(\mathbf{B})}\right)$ is isomorphic to the lattice of Figure 4 . Hence by the above $\mathcal{L}(B)$ is a subdirect product of two copies of the lattice of Figure 4.

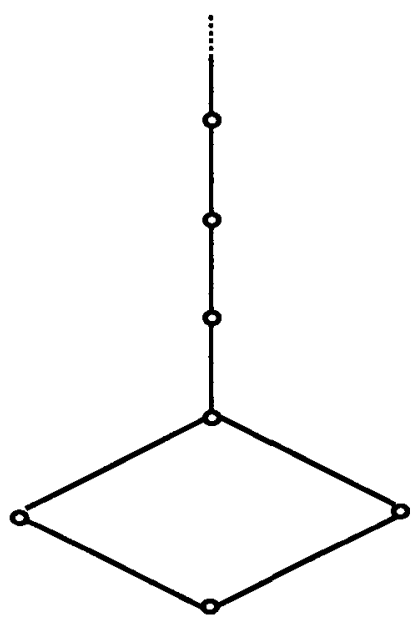

Figure 4 


\section{A complete congruence on $\mathcal{L}(\mathbf{C R})$}

If $\rho$ is any regular semigroup and $\rho$ a congruence on $S$, then we put

$$
\operatorname{ker} \rho=\left\{x \in S \mid\left(x, x^{2}\right) \in \rho\right\} .
$$

For any $\rho, \theta \in \mathcal{L}$ we shall put

$$
\rho K \theta \quad \Leftrightarrow \quad \operatorname{ker}\left(\rho / \rho_{\mathbf{C R}}\right)=\operatorname{ker}\left(\theta / \rho_{\mathbf{C R}}\right) .
$$

From [13, Theorem 4.5] the $K$-class $\rho K$ of $\rho$ is an interval $\left[\rho_{K}, \rho^{K}\right]$, and for $\rho, \theta \in \mathcal{L}$,

$$
\rho \subseteq \theta \Rightarrow \rho_{K} \subseteq \theta_{K}, \quad \rho^{K} \subseteq \theta^{K}
$$

THEOREM 11. The relation $K$ is a complete congruence on $\mathcal{L}$.

Proof. By (6), $\rho \rightarrow \rho^{K}$ is a closure operation which induces $K$ on $\mathcal{L}$, and therefore $K$ is infinitely $\vee$-compatible. Dually, by (6) $\rho \rightarrow \rho_{K}$ is a dual closure operation which induces $K$ on $\mathcal{L}$, and therefore $K$ is infinitely n-compatible.

TheOREM 12. $\mathcal{L} / K$ is a complete arguesian lattice.

Proof. For $a, b \in U(X)$ we have that $a \rho_{\mathrm{SL}} b$ if and only if $\mathbf{c}(a)=\mathbf{c}(b)$. Further $\rho_{\mathrm{SL}} / \rho_{\mathrm{CR}}$ is exactly Green's $D$-relation on the free completely regular semigroup $U(X) / \rho_{\mathbf{C R}}$. Hence $\left(a \rho_{\mathbf{C R}}\right) D\left(b \rho_{\mathbf{C R}}\right)$ if and only if $\mathbf{c}(a)=\mathbf{c}(b)$ (see also [2]). Let $\mathcal{Y}$ be the set of finite subsets of $X$. By the above we may denote the $D$-classes of $U(X) / \rho_{\mathrm{CR}}$ by $D_{Y}, Y \in \mathcal{Y}$, where $D_{Y}$ consists of the $a \rho_{\mathrm{CR}}$ for which $\mathrm{c}(a)=Y$. For each such $Y$ we choose a maximal subgroup $G_{Y}$ of $D_{Y}$.

Let $\rho \in \mathcal{L}$. Since

$$
\begin{aligned}
\operatorname{ker}\left(\rho / \rho_{\mathbf{C R}}\right) & =\operatorname{ker}\left(\rho / \rho_{\mathbf{C R}}\right) \cap \operatorname{ker}\left(\rho_{\mathbf{S L}} / \rho_{\mathbf{C R}}\right) \\
& =\operatorname{ker}\left(\left(\rho / \rho_{\mathbf{C R}}\right) \cap\left(\rho_{\mathbf{S L}} / \rho_{\mathbf{C R}}\right)\right)
\end{aligned}
$$

it follows that

$$
\operatorname{ker} \rho / \rho_{\mathbf{C R}}=\bigcup_{Y \in y}\left(\left.\operatorname{ker}\left(\rho / \rho_{\mathbf{C R}}\right)\right|_{D_{Y}}\right) \text {. }
$$

We remark that for every $Y \in \mathcal{Y},\left.\operatorname{ker}\left(\rho / \rho_{\mathbf{C R}}\right)\right|_{D_{Y}}$ is completely determined by $\left.\operatorname{ker}\left(\rho / \rho_{\mathbf{C R}}\right)\right|_{G_{Y}}=N_{Y}(\rho)$ (see e.g. in [8]).

Let $\mathcal{N}\left(G_{Y}\right)$ be the lattice of normal subgroups of $G_{Y}$. Then

$$
\psi: \mathcal{L} \rightarrow \prod_{Y \in \mathcal{Y}} \mathcal{N}\left(G_{Y}\right), \quad \rho \rightarrow\left(N_{Y}(\rho), Y \in \mathcal{Y}\right),
$$


is a well-defined mapping which induces $K$ on $\mathcal{L}$. One readily sees that for $Y \in \mathcal{Y}, \rho, \theta \in \mathcal{L}$, we have $N_{Y}(\rho \cap \theta)=N_{y}(\rho) \cap N_{y}(\theta)$, so that $\psi$ is a n-homomorphism.

By (7) and the fact that $K$ is a congruence, we have for $\rho, \theta \in \mathcal{L}$ that

$$
\operatorname{ker}(\rho \vee \theta) / \rho_{\mathbf{C R}}=\operatorname{ker}\left(\left(\rho / \rho_{\mathbf{C R}} \cap \rho_{\mathbf{S L}} / \rho_{\mathbf{C R}}\right) \vee\left(\theta / \rho_{\mathbf{C R}} \cap \rho_{\mathbf{S L}} / \rho_{\mathbf{C R}}\right)\right)
$$

whence for every $Y \in \mathcal{Y}$,

$$
\begin{aligned}
&\left.\operatorname{ker}\left((\rho \vee \theta) / \rho_{\mathbf{C R}}\right)\right|_{D_{Y}} \\
& \quad=\left.\operatorname{ker}\left(\left(\rho / \rho_{\mathbf{C R}} \cap \rho_{\mathbf{S L}} / \rho_{\mathbf{C R}}\right) \vee\left(\theta / \rho_{\mathbf{C R}} \cap \rho_{\mathbf{S L}} / \rho_{\mathbf{C R}}\right)\right)\right|_{D_{Y}} \\
& \quad=\operatorname{ker}\left(\left.\left(\left.\left(\rho / \rho_{\mathbf{C R}} \cap \rho_{\mathbf{S L}} / \rho_{\mathbf{C R}}\right)\right|_{D_{Y}}\right) \vee\left(\theta / \rho_{\mathbf{C R}} \cap \rho_{\mathbf{S L}} / \rho_{\mathbf{C R}}\right)\right|_{D_{Y}}\right) \\
& \quad=\operatorname{ker}\left(\left.\left.\left(\rho / \rho_{\mathbf{C R}}\right)\right|_{D_{Y}} \vee\left(\theta / \rho_{\mathbf{C R}}\right)\right|_{D_{Y}}\right),
\end{aligned}
$$

and it follows that

$$
N_{Y}(\rho \vee \theta)=\left.\operatorname{ker}\left(\left.\left.\left(\rho / \rho_{\mathbf{C R}}\right)\right|_{D_{Y}} \vee\left(\theta / \rho_{\mathbf{C R}}\right)\right|_{D_{Y}}\right)\right|_{G_{Y}} .
$$

From the results [8] concerning the description of congruences on completely simple semigroups, we have that

$$
\begin{aligned}
& \left.\operatorname{ker}\left(\left.\left.\left(\rho / \rho_{\mathbf{C R}}\right)\right|_{D_{Y}} \vee\left(\theta / \rho_{\mathbf{C R}}\right)\right|_{D_{Y}}\right)\right|_{G_{Y}} \\
& \quad=\left.\left.\operatorname{ker}\left(\left.\left(\rho / \rho_{\mathbf{C R}}\right)\right|_{D_{Y}}\right)\right|_{G_{Y}} \operatorname{ker}\left(\left.\left(\theta / \rho_{\mathbf{C R}}\right)\right|_{D_{Y}}\right)\right|_{G_{Y}} \\
& \quad=N_{Y}(\rho) \quad N_{Y}(\theta) .
\end{aligned}
$$

Hence $N_{Y}(\rho \vee \theta)=N_{Y}(\rho) N_{Y}(\theta)$ and we conclude that $\psi$ is a $\vee$-homomorphism.

By the above $\mathcal{L} / K$ can be isomorphically embedded into $\prod_{Y \in \mathcal{L}} \mathcal{N}\left(G_{Y}\right)$, which is an arguesian lattice. Hence, $\mathcal{L} / K$ is itself arguesian. Because of the antisomorphism $\rho \rightarrow \mathbf{V}_{\rho}$ of $\mathcal{L}$ onto $\mathcal{L}(\mathbf{C R})$, the situation described so far in this section carries over to $\mathcal{L}(\mathbf{C R})$. The complete congruence on $\mathcal{L}(\mathbf{C R})$ we obtain in this way will again be denoted by $K$.

THEOREM 13. The relation $K$ which is given by

$$
\mathbf{V} K \mathbf{W} \Leftrightarrow \mathbf{R B} \circ(\mathbf{S L} \vee \mathbf{V})=\mathbf{R B} \circ(\mathbf{S L} \vee \mathbf{W})
$$

is a complete congruence on $\mathcal{L}(\mathbf{C R})$. For $\mathbf{V} \in \mathcal{L}(\mathbf{C R})$, the $K$-class $\mathbf{V} K$ is an interval $\left[\mathbf{V}_{K}, \mathbf{V}^{K}\right]$ where $\mathbf{V}^{K}=\mathbf{R B} \circ(\mathbf{S L} \vee \mathbf{V})$.

Proof. Immediate from Theorem 11 and [9, Theorem 5.1, Proposition $6.1]$.

In $\left[9\right.$, Corollary 6.5] a set of defining identities for $\mathbf{V}^{K}=\mathbf{R B} \circ(\mathbf{S L} \vee \mathbf{V})$ is derived from a set of defining identities for $\mathbf{V}$. From [9, Proposition 7.2] 
we know that $\mathbf{V}^{K}$ is, in Reilly's notation [19], $(\mathrm{SL} \vee \mathrm{V})^{p}$. The variety $\mathbf{V}^{K}$ was denoted by $\mathbf{V}_{\left(\rho_{V}\right)^{\min }}$ in [13]. We now point out the connection between the above results and the results of [16].

Obviously, if $\rho \in \mathcal{L}$, then $\rho_{K}$ is the fully invariant congruence associated with $\left(\mathrm{V}_{\rho}\right)^{K}$. More details are given by the following.

THEOREM 14. For $\rho \in \mathcal{L}$, the relation $\rho_{K}$ can be defined inductively by $a \rho_{K} b \Leftrightarrow a\left(\rho \cap \rho_{\mathrm{SL}}\right) b, \quad \mathbf{s}(a) \rho_{K} \mathbf{s}(b), \quad \mathbf{d}(a) \rho_{K} \mathbf{d}(b) \quad(a, b \in U(X))$.

Proof. Let $\theta$ be defined inductively by

$$
a \theta b \Leftrightarrow a\left(\rho \cap \rho_{\mathrm{SL}}\right) b, \quad \mathbf{s}(a) \rho_{K} \mathbf{s}(b), \quad \mathbf{d}(a) \rho_{K} \mathbf{d}(b) .
$$

By [16, Theorem $1(1)], \theta \in \mathcal{L}$. Obviously $\theta \subseteq \rho$, hence $\operatorname{ker}\left(\theta / \rho_{\mathbf{C R}}\right) \subseteq$ $\operatorname{ker}\left(\rho / \rho_{\mathbf{C R}}\right)$. If $b \in \operatorname{ker}\left(\rho / \rho_{\mathbf{C R}}\right)$ then

$$
b\left(\rho \cap \rho_{\mathrm{SL}}\right) b(b)^{-1}, \quad \mathbf{s}(b)=\mathbf{s}\left(b(b)^{-1}\right), \quad \mathbf{d}(b)=\mathbf{d}\left(b(b)^{-1}\right),
$$

hence $b \theta b(b)^{-1}$ and so $b \in \operatorname{ker}\left(\theta / \rho_{\mathrm{CR}}\right)$. We conclude that $\operatorname{ker}\left(\rho / \rho_{\mathrm{CR}}\right)=$ $\operatorname{ker}\left(\theta / \rho_{\mathrm{CR}}\right)$, whence $\rho K \theta$ and $\rho_{K} \subseteq \theta \subseteq \rho$.

Let $a, b \in U(X)$, such that $a(a)^{-1} \theta b(b)^{-1}$. Then

$$
\mathbf{s}\left(a(a)^{-1}\right) \rho_{K} \mathbf{s}\left(b(b)^{-1}\right)
$$

and by $[2, \S 5]$

$$
\begin{aligned}
& \left(a(a)^{-1}\right) \rho_{\mathbf{C R}} R\left(\mathbf{s}\left(a(a)^{-1}\right) x\right) \rho_{\mathbf{C R}}, \\
& \left(b(b)^{-1}\right) \rho_{\mathbf{C R}} R\left(\mathbf{s}\left(b(b)^{-1}\right) x\right) \rho_{\mathbf{C R}}
\end{aligned}
$$

in $U(X) / \rho_{\mathbf{C R}}$, with $\{x\}=\mathbf{c}(a)-\mathbf{c}(\mathbf{s}(a))=\mathbf{c}(b)-\mathbf{c}(\mathbf{s}(b))$. We infer that

$$
\left(a(a)^{-1}\right) \rho_{K} R\left(b(b)^{-1}\right) \rho_{K}
$$

in $U(X) / \rho_{K}$. This result and its dual now entails that $a(a)^{-1} \rho_{K} b(b)^{-1}$. Since $\rho_{K} \subseteq \theta$ we can now conclude that

$$
a(a)^{-1} \rho_{K} b(b)^{-i} \Leftrightarrow a(a)^{-1} \theta b(b)^{-1} .
$$

In view of [10, Corollary 7] we can now conclude that $\theta=\rho_{K}$.

From Theorems 11 and 14 now follows Theorem 1(3), (4) of [16]. Our relation $K$ is denoted by $\rho$ in [16].

\section{A subdirect decomposition of $\mathcal{L}(\mathbf{C R})$}

We are now able to prove one of the main results of this paper. 
THEOREM 15. The mapping

$$
\psi: \mathbf{V} \rightarrow\left(\mathbf{V} T_{l}, \mathbf{V} K, \mathbf{V} T_{r}\right), \quad \mathbf{V} \in \mathcal{L}(\mathbf{C R}),
$$

is an isomorphism of $\mathcal{L}(\mathbf{C R})$ onto a subdirect product of $\mathcal{L}(\mathbf{C R}) / T_{l}, \quad \mathcal{L}(\mathbf{C R}) / K$ and $\mathcal{L}(\mathbf{C R}) / T_{r}$.

Proof. The above considered map $\psi$ is certainly a homomorphism by Theorems 9 and 13. Further,

$$
\begin{aligned}
\mathbf{V} \psi=\mathbf{W} \psi & \Leftrightarrow \rho_{\mathbf{v}}\left(T_{l} \cap K \cap T_{r}\right) \rho_{\mathbf{W}} \\
& \Leftrightarrow \rho_{\mathbf{v}}=\rho_{\mathbf{W}} \quad \text { by [10, Theorem 26] } \\
& \Leftrightarrow \mathbf{V}=\mathbf{W} .
\end{aligned}
$$

We conclude that $\psi$ is injective.

Theorem 15 corresponds with Theorem 26 of [10] and is, by Lemma 7 and Theorem 14, concordant with Theorem 3(3) of [16].

\section{On the modularity of $\mathcal{L}(\mathrm{CR})$}

Let $\Lambda$ be the poset of Figure 5 and let $\Delta$ be the ordinal sum of the poset of Figure 6 and $\mathcal{L}(\mathbf{C R}) / K$. Then $\Delta$ is a complete arguesian lattice by Theorem

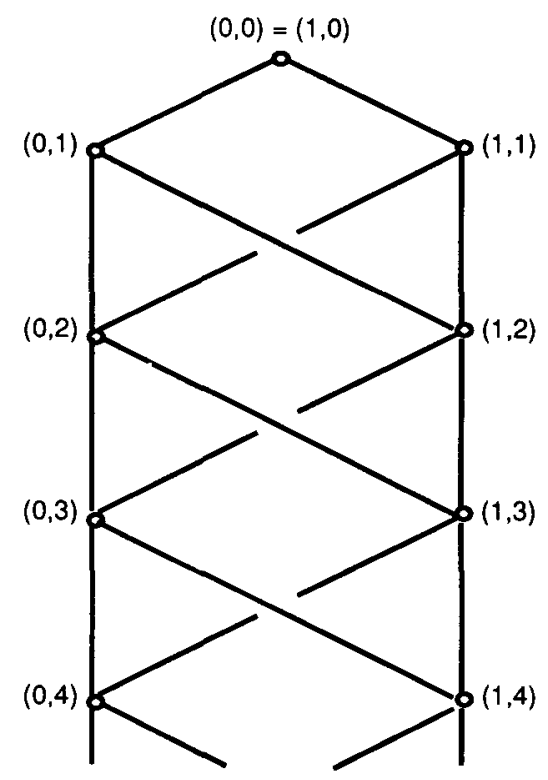

Figure 5 


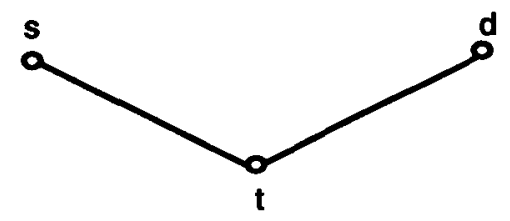

Figure 6

12. Hence the poset of all isotone mappings of $\Lambda$ into $\Delta$ forms a complete arguesian lattice $\Gamma$. We shall show that $\mathcal{L}(\mathbf{C R})$ can be isomorphically embedded into the lattice $\Gamma$.

For $\mathbf{V} \in \mathcal{L}(\mathbf{C R})$ we put

$$
\begin{aligned}
& \mathbf{V}(0,0)=\mathbf{V}(1,0)=\mathbf{V} \vee \mathbf{S L}, \\
& \mathbf{V}(0, n+1)=\mathbf{V}(1, n)_{T_{r}}, \\
& \mathbf{V}(1, n+1)=\mathbf{V}(0, n)_{T_{i}}
\end{aligned}
$$

For $\mathrm{V} \in \mathcal{L}(\mathbf{C R})$ and $(i, n), \quad i=0,1, n \in \mathbf{N}$, we put

$$
\xi_{\mathbf{V}}(i, n)= \begin{cases}\mathbf{s} & \text { if } i=0, n \geq 1 \text { and } \mathbf{V}(0, n)=\mathbf{L N B}, \\ \mathbf{d} & \text { if } i=1, n \geq 1 \text { and } \mathbf{V}(1, n)=\mathbf{R N B}, \\ \mathbf{T} & \text { if } n \geq 1 \text { and } \mathbf{V}(i, n)=\mathbf{S L}, \\ \mathbf{V}(i, n) K & \text { otherwise. }\end{cases}
$$

By Lemmas 6 and 7, the last theorem of [16] can be reformulated as follows.

RESULT 16 [16]. The mapping $\mathrm{V} \rightarrow \xi_{\mathrm{V}}$ is an order isomorphism of the interval [SL, CR] into the lattice $\Gamma$ of isotone mappings of $\Lambda$ into $\Delta$.

LEMMA 17. The mapping $\mathrm{V} \rightarrow \xi_{\mathrm{v}}$ is an injective homomorphism of [SL, CR] into $\Gamma$.

Proof. Let $\mathbf{V}, \mathbf{W} \in[\mathbf{S L}, \mathbf{C R}]$. We must show that

$$
\xi_{\mathrm{v} \vee \mathrm{w}}(i, n)=\xi_{\mathrm{v}}(i, n) \vee \xi_{\mathrm{w}}(i, n)
$$

and

$$
\xi_{\mathbf{v} \cap \mathbf{W}}(i, n)=\xi_{\mathbf{v}}(i, n) \wedge \xi_{\mathbf{W}}(i, n)
$$

for all $(i, n)$.

Obviously (8) and (9) are satisfied if $n=0$ by Theorem 13 . We next assume that $n>0$ and we verify (8) and (9) in case $i=0$. We have that

$$
\mathbf{V}(0, n)=\mathbf{V}(1, n-1)_{T_{r}}, \quad \mathbf{W}(0, n)=\mathbf{W}(1, n-1)_{T_{r}},
$$


where both $\mathbf{V}(0, n)$ and $\mathbf{W}(0, n)$ contain SL. According to Lemma 5 , the following cases can occur:

(i) $\mathbf{S L}=\mathbf{V}(0, n) \subseteq \mathbf{V}(1, n-1) \subseteq \mathbf{R G} \circ \mathbf{S L}$ and then $\xi_{\mathbf{V}}(0, n)=\mathbf{t}$,

(ii) $\mathbf{L N B}=\mathbf{V}(0, n) \subseteq \mathbf{V}(1, n-1) \subseteq \mathbf{R G} \circ \mathbf{L N B}$ and then $\xi_{\mathrm{V}}(0, n)=\mathbf{s}$,

(iii) $\quad \mathbf{L R B} \subseteq \mathrm{V}(0, n) \subseteq \mathrm{V}(1, n-1)$ and then $\xi_{\mathrm{V}}(0, n)=\mathrm{V}(0, n) K$,

(a) $\mathbf{S L}=\mathbf{W}(0, n) \subseteq \mathbf{W}(1, n-1) \subseteq \mathbf{R G} \circ \mathbf{S L}$ and then $\xi_{\mathbf{W}}(0, n)=\mathbf{t}$,

(b) $\mathbf{L N B}=\mathbf{W}(0, n) \subseteq \mathbf{W}(1, n-1) \subseteq \mathbf{R G} \circ \mathbf{L N B}$ and then $\xi_{\mathbf{W}}(0, n)=\mathbf{s}$,

(c) $\quad \mathbf{L R B} \subseteq \mathbf{W}(0, n) \subseteq \mathbf{W}(1, n-1)$ and then $\xi_{\mathbf{W}}(0, n)=\mathbf{W}(0, n) K$.

This leaves us with nine possible cases to consider.

By Theorem 9 and its dual we have that

$$
\mathbf{V}(i, k) \cap \mathbf{W}(i, k)=(\mathbf{V} \cap \mathbf{W})(i, k)
$$

and

$$
\mathbf{V}(i, k) \vee \mathbf{W}(i, k)=(\mathbf{V} \vee \mathbf{W})(i, k),
$$

for $i=0,1$ and $k \geq 1$.

Assume first that (iii) and (c) hold. Then

$$
\mathbf{L R B} \subseteq \mathbf{V}(0, n) \cap \mathbf{W}(0, n)=(\mathbf{V} \cap \mathbf{W})(0, n),
$$

and

$$
\mathbf{L R B} \subseteq \mathbf{V}(0, n) \vee \mathbf{W}(0, n)=(\mathbf{V} \vee \mathbf{W})(0, n)
$$

It follows that

$$
\begin{aligned}
\xi_{\mathbf{V}}(0, n) \wedge \xi_{\mathbf{W}}(0, n) & =\mathbf{V}(0, n) K \cap \mathbf{W}(0, n) K \\
& =(\mathbf{V}(0, n) \cap \mathbf{W}(0, n)) K \quad(\text { by Theorem 13) } \\
& =((\mathbf{V} \cap \mathbf{W})(0, n)) K \quad(\text { by }(10)) \\
& =\xi_{V \cap W}(0, n)
\end{aligned}
$$

and similarly (8) holds.

Assume next that (iii) and (b) hold. Then again

$$
\mathbf{L R B} \subseteq \mathbf{V}(0, n) \vee \mathbf{W}(0, n)=(\mathbf{V} \vee \mathbf{W})(0, n),
$$

whence

$$
\begin{aligned}
& \xi_{\mathbf{V}}(0, n) \vee \xi_{\mathbf{W}}(0, n)=\mathbf{V}(0, n) K \vee \mathbf{s}=\mathbf{V}(0, n) K \\
& =\quad \mathbf{V}(0, n) K \vee \mathbf{W}(0, n) K \\
& \quad(\text { since } \mathbf{W}(0, n) K \text { is the least element of } \mathcal{L}(\mathbf{C R}) / K) \\
& =(\mathbf{V}(0, n) \vee \mathbf{W}(0, n)) K \quad(\text { by Theorem 13) } \\
& =((\mathbf{V} \vee \mathbf{W})(0, n)) K \quad(\text { by }(11)) \\
& =\xi_{\mathrm{V} \vee \mathbf{W}}(0, n) .
\end{aligned}
$$


Further,

$$
\mathbf{L N B}=\mathbf{W}(0, n)=\mathbf{V}(0, n) \cap \mathbf{W}(0, n)=(\mathbf{V} \cap \mathbf{W})(0, n)
$$

and therefore

$$
\xi_{\mathrm{V}}(0, n) \wedge \xi_{\mathrm{W}}(0, n)=\mathbf{V}(0, n) K \wedge \mathrm{s}=\mathbf{s}=\xi_{\mathrm{V} \cap \mathbf{W}}(0, n) .
$$

The remaining cases can be treated in a similar way. We conclude that (8) and (9) hold for all $n$ if $i=0$. The case $i=1$ can be handled in a dual way.

THEOREM 18. $\mathcal{L}(\mathbf{C R})$ is an arguesian lattice.

Proof. It follows from Lemma 17 that [SL, CR] is arguesian. From [7, Proposition 3.5] we know that

$$
\mathcal{L}(\mathbf{C R}) \rightarrow[\mathbf{S L}, \mathbf{C R}] \times[\mathbf{T}, \mathbf{S L}], \quad \mathrm{V} \rightarrow(\mathrm{V} \vee \mathbf{S L}, \mathbf{V} \cap \mathbf{S L})
$$

is an injective homomorphism. Thus $\mathcal{L}(\mathbf{C R})$ is arguesian.

Since $\mathcal{L}(\mathbf{C R})$ is arguesian, it follows that $\mathcal{L}(\mathbf{C R})$ is modular. The modularity of several particular sublattices of $\mathcal{L}(\mathbf{C R})$ was established in [1], [3], [4], [5], [7], [17], [18].

\section{References}

[1] A. P. Birjukov, 'Varieties of idempotent semigroups', Algebra i Logika 9 (1970), 255273. (in Russian).

[2] A. H. Clifford, 'The free completely regular semigroup on a set', J. Algebra 59 (1979), 434-451.

[3] C. F. Fennemore, 'All varieties of bands I', Math. Nachr. 48 (1971), 237-252, II, Math. Nachr. 48 (1971), 253-262.

[4] J. A. Gerhard, 'The lattice of equational classes of idempotent semigroups', J. Algebra 15 (1979), 195-224.

[5] J. A. Gerhard and M. Petrich, 'All varieties of regular orthogroups', Semigroup Forum 31 (1985), 311-351.

[6] G. Grätzer, General lattice theory, Birkhaüser, Basel, 1978.

[7] T. E. Hall and P. R. Jones, 'On the lattice of varieties of bands of groups', Pacific J. Math. 91 (1980), 327-337.

[8] J. M. Howie, An introduction to semigroup theory, Academic Press, London, 1976.

[9] P. R. Jones, 'Mal' cev products of varieties of completely regular semigroups', J. Austral Math. Soc. (Series A) 42 (1987), 227-246.

[10] F. Pastijn, Congruences on regular semigroups-A survey, in Proceedings of the 1984 Marquette Conference on Semigroups, (K. Byleen, P. Jones, F. Pastijn, eds.), Marquette University, 1985, pp. 159-175

[11] F. Pastijn and M. Petrich, 'Congruences on regular semigroups', Trans. Amer. Math. Soc. 295 (1986), 607-633. 
[12] F. Pastijn and M. Petrich, 'The congruence lattice of a regular semigroup', J. Pure Appl. Algebra 53 (1988), 93-123.

[13] F. Pastijn and P. G. Trotter, 'Lattices of completely regular semigroup varieties', Pacific J. Math 119 (1985), 191-214.

[14] M. Petrich, Lectures in semigroups, Wiley, London, 1977.

[15] M. Petrich, 'The structure of completely regular semigroups', Trans. Amer. Math. Soc. 189 (1974), 211-236.

[16] L. Polák, 'On varieties of completely regular semigroups I', Semigroup Forum 32 (1985), 97-123.

[17] V. V. Rasin, 'On the varieties of Cliffordian semigroups', Semigroup Forum 23 (1981), 201-220.

[18] V. V. Rasin, 'Varieties of orthodox Clifford semigroups', Izv. Vyssh. Uchebn. Zaved. Mat. 26 (1982), 82-85 (in Russian).

[19] N. R. Reilly, 'Varieties of completely regular semigroups', J. Austral. Math. Soc. (Series A) 38 (1985), 372-393.

Department of Mathematics, Statistics

and Computer Science,

Marquette University,

Milwaukee, Wisconsin 53233

U.S.A. 